\title{
Berezinskii-Kosterlitz-Thouless transitions in the six-state clock model
}

\author{
Haruhiko Matsuo and Kiyohide Nomura \\ Department of Physics, Kyushu University \\ Fukuoka 812-8581 JAPAN \\ halm@stat.phys.kyushu-u.ac.jp \\ knomura@stat.phys.kyushu-u.ac.jp
}

June 19, 2021

\begin{abstract}
Classical 2D clock model is known to have a critical phase with Berezinskii-Kosterlitz-Thouless(BKT) transitions. These transitions have logarithmic corrections which make numerical analysis difficult. In order to resolve this difficulty, one of the authors has proposed the method called level spectroscopy, which is based on the conformal field theory. We extend this method to the multi-degenerated case. As an example, we study the classical 2D 6-clock model which can be mapped to the quantum self-dual 1D 6-clock model. Additionally, we confirm that the self-dual point has a precise numerical agreement with the analytical result, and we argue the degeneracy of the excitation states at the self-dual point from the effective field theoretical point of view.
\end{abstract}

\section{Introduction}

Although low dimensional spin systems with a continuous rotational symmetry, like the 2D XY spin model, don't have a symmetry breaking phase at finite temperatures, it doesn't mean the absence of phase transitions. Actually Berezinskii [1] and Kosterlitz and Thouless [2] pointed out the vortexantivortex excitation phase transition in the 2D XY model, which is known as the BKT transition. 
The classical 2D clock model, which has a discrete rotational symmetry, is also known to have a BKT critical region. José et al 3 s studied the 2D classical $p$-state clock model by the renormalization group analysis and pointed out the presence of the intermediate critical phase for large $p$. Elitzur et al [4] have also obtained the same result through the correlation inequality argument.

We map the 2D p-clock model onto its quantum 1D p-clock model and analyze the latter. This is based on the fact that the $(1+d)$ dimensional classical statistical mechanics can be mapped onto the $d$-dimensional quantum statistical mechanics. Here, we should be careful that the 2D classical clock model, introduced the following section, doesn't have the self-duality explicitly, although its quantized Hamiltonian has the self-duality. It may seems strange, but thinking of the universality class; that is, the $Z_{p}$ Villain model and the $2 \mathrm{D}$ classical $p$-clock model are considered belong to the same universality class, the self-duality of the quantum clock model is acceptable. Additionally, the corresponding continuum model(the dual sine-Gordon model) also has the self-duality. We will see an excellent numerical agreement with the self-duality.

Generally, the BKT transition points were difficult to determine numerically because of the logarithmic corrections and the anomalous divergence of the correlation length. But the level spectroscopy method [5] [6] has been proposed to resolve these problems. Near a BKT transition point, some scaling dimensions change from relevant to irrelevant or vice versa. Choosing some applicable scaling dimensions, we can determine the BKT transition point by the crossing point of these scaling dimensions. In addition, we can eliminate logarithmic corrections accompanying the BKT transition. This is the brief explanation of the level spectroscopy.

So far the level spectroscopy has been applied to the three cases. The first case is between the BKT critical phase and the phase of the non-degenerate ground state [6]. The second is between the BKT critical phase and the phase of 2-fold degenerate ground state [5. The third is between the BKT critical phase and the multi-degenerated ground state phase. Recently Tonegawa et al [8] studied the $1 / 3$ plateau problem of the $S=\frac{1}{2}$ antiferromagnetic XXZ chain with the $Z_{3}$ symmetry breaking and Otsuka et al[?] studied the $2 \mathrm{D}$ AF 3 -state Potts model with the $Z_{6}$ symmetry breaking case.

In this paper, we apply the level spectroscopy to the $1 \mathrm{D}$ quantum 6-clock model with duality and use the duality relation to check the level spectroscopy results between the BKT critical phase and the multi-degenerated ground state from another point of view than former studies. In addition, we 
discuss the degeneracy at the self-dual point from the field theoretical point of view. The self-dual point of this model is trivial but it will be important in the 2D AF 3-state Potts model which have no explicit self-duality.

\section{Theory}

\section{$2.1 \quad$ Discrete model}

In this subsection, we review the $1 \mathrm{D}$ quantum $p$-state clock model with a nearest neighbor interaction as a Hamiltonian limit of a $2 \mathrm{D}$ classical $p$-state clock model on a square lattice. The 2D classical Hamiltonian is

$$
\begin{aligned}
\mathcal{H}= & -\beta \sum_{\langle i, j\rangle}\left\{J_{s}\left[\cos \left(\Theta_{i, j}-\Theta_{i+1, j}\right)-1\right]\right. \\
& \left.+J_{\tau}\left[\cos \left(\Theta_{i, j}-\Theta_{i, j+1}\right)\right]\right\}
\end{aligned}
$$

where $\Theta_{i, j}=(2 \pi r / p), r=0,1, \ldots, p-1$ is the clock spin variable. When we take the Hamiltonian limit $\beta J_{s} \rightarrow 0, \beta J_{\tau} \rightarrow \infty$ with $\lambda$ fixed[10], we obtain the 1D quantum Hamiltonian [4] 10]

$$
H=-2 \sum_{n=1}^{N}\left\{\lambda \cos \left(\hat{\Theta}_{n}-\hat{\Theta}_{n+1}\right)+\cos \hat{p}_{n}\right\}
$$

where $\lambda$ is the transverse field of the system. It can be interpreted as the inverse of the temperature.

When we define the operators $\sigma_{n}, \Gamma_{n}$ as $\sigma_{n}=\exp i \hat{\Theta}, \Gamma_{n}=\exp i \hat{p}$, we obtain another representation of the 1D quantum Hamiltonian (2) as

$$
H=-\sum_{n=1}^{L}\left\{\lambda\left(\sigma_{n} \sigma_{n+1}^{+}+\sigma_{n}^{+} \sigma_{n+1}\right)+\Gamma_{n}+\Gamma_{n}^{+}\right\}
$$

where $\omega=\exp (2 \pi i / p)$, and

$$
\sigma_{n}=\left(\begin{array}{ccccc}
1 & & & & \\
& \omega & & & \\
& & \omega^{2} & & \\
& & \ddots & \\
& & & & \omega^{p-1}
\end{array}\right), \quad \Gamma_{n}=\left(\begin{array}{ccccc}
0 & & & & 1 \\
1 & 0 & & & \\
& 1 & 0 & & \\
& & \ddots & \ddots & \\
& & & 1 & 0
\end{array}\right) .
$$


These operators satisfy $\left(\sigma_{n}\right)^{p}=\left(\Gamma_{n}\right)^{p}=1$.

This model has a $Z_{p}$ charge symmetry. The $Z_{p}$ charge operator is defined as

$$
U_{Q}=\prod_{n=1}^{L} \Gamma_{n}
$$

which commutes with the Hamiltonian (3). $U_{Q}$ has eigenvalues $\omega^{Q}, Q=$ $0,1, \cdots, p-1$. Because of the conservation, $U_{Q}$ splits $H$ into the charge sectors whose corresponding eigenspaces are labeled by $Q=0,1, \cdots, p-1$, i.e. $H$ is able to be block-diagonalized. Also we can block-diagonalize it with the toroidal boundary conditions:

$$
\sigma_{N+1}=\exp \left(\frac{2 \pi i}{p} \tilde{Q}\right) \sigma_{1}, \tilde{Q}=0,1, \ldots, p-1 .
$$

$\tilde{Q}=0$ is the periodic boundary condition and $\tilde{Q}=p / 2(p$ even $)$ is the twisted boundary condition. Hereafter we will assume that $p$ is even. Implementing the twisted boundary condition to the system corresponds to adding a halfcharge to the sine-Gordon model[7] [1] which is the continuum limit of the discrete model.

There exits a unitary operator such that $\sigma_{n}$ is transformed into $\Gamma_{n}^{+}$and also $\Gamma_{n}$ is transformed into $\sigma_{n}$. For example we can take its matrix elements as

$$
U_{i, j}=\frac{1}{\sqrt{p}} \omega^{(1-i)(1-j)} .
$$

This operator $U$ gives the relation

$$
\begin{aligned}
& U \sigma_{n} U^{+}=\Gamma_{n}^{+}, \\
& U \Gamma_{n} U^{+}=\sigma_{n} .
\end{aligned}
$$

By this relation, we get another representation of the 1D quantum Hamiltonian as

$$
H^{\prime}=-\sum_{n=1}^{L}\left\{\lambda\left(\Gamma_{n} \Gamma_{n+1}^{+}+\Gamma_{n}^{+} \Gamma_{n+1}\right)+\sigma_{n}+\sigma_{n}^{+}\right\}
$$


In this case, notice that the $Z_{p}$ charge operator should be

$$
U_{Q}^{\prime}=\prod_{n=1}^{L} \sigma_{n}
$$

The form (9), (10) is more useful for numerical calculation than the pribious form (3), when we choose the $\sigma_{n}$ diagonal representation.

There is another non-local transformation between $\sigma$ and $\Gamma$ :

$$
\begin{aligned}
\sigma_{n} & \rightarrow \tilde{\sigma}_{n}=\Gamma_{n+1} \Gamma_{n}^{+} \quad \text { for } n<N \\
\sigma_{N} & \rightarrow \tilde{\sigma}_{N}=\Gamma_{N}^{+} \\
\Gamma_{n} & \rightarrow \tilde{\Gamma}_{n}=\prod_{l=1}^{n} \sigma_{l} .
\end{aligned}
$$

Applying this transformation to the Hamiltonian (91) and the help of

$$
\tilde{\Gamma}_{n} \tilde{\Gamma}_{n+1}^{+}=\prod_{l=1}^{n} \sigma_{l} \prod_{l=1}^{n+1} \sigma_{l}^{+}=\sigma_{n+1}^{+},
$$

we get the dual form of (9) as

$$
-\sum_{n=1}^{L}\left[\lambda\left(\sigma_{n}^{+}+\sigma_{n}\right)+\Gamma_{n} \Gamma_{n+1}^{+}+\Gamma_{n}^{+} \Gamma_{n+1}\right] .
$$

Therefore the 1D p-state quantum clock model satisfies the following duality relation,

$$
H(\lambda)=\lambda H(1 / \lambda)
$$

The self-dual point is $\lambda_{c}=1$.

The charge conjugation operator is defined as

$$
C=\prod_{n=1}^{L} c_{n}, \quad c_{n}=\left(\begin{array}{ccccc}
1 & & & & 0 \\
& 0 & & 0 & 1 \\
& & \ddots & 1 & \\
& 0 & 1 & \ddots & \\
0 & 1 & & & 0
\end{array}\right)
$$


which has eigenvalues \pm 1 and transforms $\sigma_{n}$ and $\Gamma_{n}$ as

$$
\begin{aligned}
& c_{n} \sigma_{n} c_{n}^{+}=\sigma_{n}^{+} \\
& c_{n} \Gamma_{n} c_{n}^{+}=\Gamma_{n}^{+} .
\end{aligned}
$$

By this relation, it is easy to see that the Hamiltonian (3) and (9) commutes with $C$.

$Z_{p}$ charge operator $U_{Q}$ satisfies

$$
C U_{Q} C=U_{Q}^{+}
$$

So the eigenvalues $\omega^{Q}$ of $U_{Q}$ become $\omega^{-Q}$, that is, generally the eigenstates of $U_{Q}$ are not those of $C$. But the eigenstates of $Q=0, p / 2$ are invariant under this transformation, therefore these eigenstates have eigenvalues $C= \pm 1$. We will use this fact to classify the eigenstates.

\subsection{Effective model}

As an effective theory of a 1D quantum spin system, the sine-Gordon model has been studied. Here, we will explain the $Z_{p}$ dual sine-Gordon model[12],

$$
\mathcal{L}=\frac{1}{2 \pi K}(\nabla \phi)^{2}+\frac{y_{\phi}}{2 \pi \alpha^{2}} \cos \sqrt{2} \phi+\frac{y_{\theta}}{2 \pi \alpha^{2}} \cos p \sqrt{2} \theta,
$$

where $K$ is also inverse of temperature as $\lambda, \alpha$ is an ultraviolet cutoff and $\phi$ and $\theta$ are periodic:

$$
\begin{aligned}
\phi & =\phi+\frac{2 \pi}{\sqrt{2}}, \\
\theta & =\theta+\frac{2 \pi}{\sqrt{2}} .
\end{aligned}
$$

The field $\theta$ is dual to $\phi$ and they satisfy the relation

$$
\partial_{x} \phi=-\partial_{y}(K \theta), \quad \partial_{y} \phi=\partial_{x}(K \theta) .
$$

This model is the effective model for the Hamiltonian (3), (9).

The vertex operators are defined as

$$
O_{m, n}=\exp (i m \sqrt{2} \theta) \exp (i n \sqrt{2} \phi) .
$$


When $y_{\phi}=y_{\theta}=0$, its scaling dimension is

$$
x_{m, n}(K)=\frac{1}{2}\left(n^{2} K+\frac{m^{2}}{K}\right) .
$$

In a high temperature region ( $K$ small) the second term of (19) is more relevant than the third term. So the renormalization group behaviour in the high temperature region can be studied through the Lagrangian

$$
\mathcal{L}_{\text {high }}=\frac{1}{2 \pi K}(\nabla \phi)^{2}+\frac{y_{\phi}}{2 \pi \alpha^{2}} \cos \sqrt{2} \phi .
$$

When $K=4$ this sine-Gordon model shows the BKT transition, and that is studied by the level spectroscopy [7]. On the other hand, in the low temperature region the second term of (19) is more irrelevant than the third term. Then we deal with the sine-Gordon Lagrangian

$$
\mathcal{L}_{\text {low }}=\frac{K}{2 \pi}(\nabla \theta)^{2}+\frac{y_{\theta}}{2 \pi \alpha^{2}} \cos p \sqrt{2} \theta
$$

with the $Z_{p}$ symmetry breaking term. Here we got the first term through substituting (19) to (22).

Under the change of the cutoff $\alpha \rightarrow e^{l} \alpha$ and parameterizing $K / 4=$ $1+y_{0} / 2$, the renormalization group equations for (25) are

$$
\frac{d y_{0}(l)}{d l}=-y_{\phi}^{2}(l), \quad \frac{d y_{\phi}(l)}{d l}=-y_{\phi}(l) y_{0}(l)
$$

where $l$ is related to $e^{l}=L$ for the finite system size. For the case (26) $)$, by parameterizing $K^{-1} p^{2} / 4=1+y_{0} / 2$ and using $y_{\theta}$ instead of $y_{\phi}$, we get the renormalization equations

$$
\frac{d y_{0}(l)}{d l}=-y_{\theta}^{2}(l), \quad \frac{d y_{\theta}(l)}{d l}=-y_{\theta}(l) y_{0}(l)
$$

In the high temperature limit $\lambda \rightarrow 0$ (or $K \rightarrow 0$ ) this model has a nondegenerated ground state. The transition point between the non-degenerated ground state phase and the BKT critical phase can be determined by the $K=4$ version of the level spectroscopy. In this case, the renormalized scaling dimensions are given in [7]. In the low temperature limit $\lambda \rightarrow \infty$, the model has a $p$-fold degenerated ground state. In this case, the simple $K=4$ version of the level spectroscopy is no longer available. The BKT 
Table 1: Operator content of the $K=p^{2} / 4$ sine-Gordon model with $Z_{p}$ symmetry breaking term. TBC is short for twisted boundary condition. Others are under periodic boundary condition.

\begin{tabular}{|c|c|c|c|c|c|}
\hline \multirow[t]{2}{*}{ field } & \multicolumn{2}{|c|}{ quantum number } & \multirow[t]{2}{*}{ B.C. } & \multirow{2}{*}{$\begin{array}{c}\text { scaling } \\
\text { dimension } \\
x_{m, n}\end{array}$} & \multirow{2}{*}{$\begin{array}{c}\text { renormalized } \\
\text { scaling } \\
\text { dimension } \\
\end{array}$} \\
\hline & $Q$ & $C$ & & & \\
\hline $\exp (i m \sqrt{2} \theta)$ & $m \neq p / 2, p$ & & $\mathrm{PBC}$ & $x_{m, 0}$ & $\frac{2 m^{2}}{p^{2}}\left(1+\frac{y_{0}}{2}\right)$ \\
\hline $\cos (\sqrt{2} p \theta / 2)$ & $p / 2$ & 1 & $\mathrm{PBC}$ & $x_{p / 2,0}$ & $\frac{1}{2}\left(1-\frac{3}{2} y_{0}\right)$ \\
\hline $\sin (\sqrt{2} p \theta / 2)$ & $p / 2$ & -1 & $\mathrm{PBC}$ & $x_{p / 2,0}$ & $\frac{1}{2}\left(1+\frac{1}{2} y_{0}\right)$ \\
\hline \multirow[t]{2}{*}{ marginal } & $p$ & 1 & $\mathrm{PBC}$ & $x_{p, 0}$ & \\
\hline & 0 & 1 & PBC & $x_{0,1}$ & $\frac{9}{2}\left(1-\frac{1}{2} y_{0}\right)$ \\
\hline $\cos (\sqrt{2} \phi / 2)$ & 0 & 1 & TBC & $x_{0, \frac{1}{2}}$ & $\frac{9}{8}\left(1-\frac{1}{2} y_{0}\right)$ \\
\hline $\sin (\sqrt{2} \phi / 2)$ & 0 & -1 & TBC & $x_{0, \frac{1}{2}}$ & \\
\hline
\end{tabular}

critical transition point can be determined by the $K=p^{2} / 4$ version of the level spectroscopy (see also [6] and [?]). The renormalized scaling dimensions of near $K=p^{2} / 4$ are shown in Tabld(note that implementing TBC is the same as considering the half-charge of the effective model [11]).

Assuming conformal invariance, the scaling dimensions $x_{m, n}$ are related to the energy gap of the finite size system with periodic boundary conditions as

$$
x_{m, n}=\frac{L}{2 \pi v}\left(E_{m, n}(L)-E_{\mathrm{g}}(L)\right),
$$

where $L$ is the system size, $E_{\mathrm{g}}(L)$ is the ground state energy, and $v$ is the velocity of the system[13]. And the central charge $c$, which we will use to confirm the universality class of the system, is given by the finite size correction of the system as

$$
E_{\mathrm{g}}(L)=e_{\mathrm{g}} L-\frac{\pi v c}{6 L}
$$

where $e_{\mathrm{g}}$ is the bulk ground state energy per site [14].

At last, we discuss the dual transformation in this dual sine-Gordon model, corresponding with the discrete case (11), (14). When we apply the dual transformation

$$
\phi \rightarrow p \theta, \quad p \theta \rightarrow \phi
$$


to the dual sine-Gordon model (19), we get the dual form

$$
\mathcal{L}_{\text {dual }}=\frac{p^{2}}{2 \pi K}(\nabla \theta)^{2}+\frac{y_{\phi}}{2 \pi \alpha^{2}} \cos p \sqrt{2} \theta+\frac{y_{\theta}}{2 \pi \alpha^{2}} \cos \sqrt{2} \phi .
$$

When we substitute (22) to (32), we obtain the same form as (19) aside the coefficients; assuming $y_{\phi}=y_{\theta}$ and $p=K$, including the coefficients, we get the same equation as (19). This is the self-dual point of the dual sineGordon model. Note that with the dual transformation (31), fields in Table [1 are exchanged as $\cos \sqrt{2} p \theta / 2 \leftrightarrow \cos \sqrt{2} p / 2$ etc. These exchanges mean the degeneracy of the excitation spectra at the self-dual point.

The quantum discrete model has the self-dual point(14), and now this self-duality is extended to the continuum model. In the following section, we numerically estimate the self-dual point with good precision.

\section{Numerical Results}

In the previous section we have argued the general $p$-state clock model. In this section we show the numerical results for the $p=6$ case. The ground states with the various $Z_{p}$ charges and the boundary conditions are obtained by the Lanczos method. The system sizes are up to $L=10$.

First, we determine the two BKT transition points. These points have essentially different property, because one is located between the non-degenerate ground state phase and the BKT critical phase, the another is located between the 6-fold degenerate ground states phase and the BKT critical phase.

Next, to confirm the universality class of the BKT critical phase, we calculate the ratio of the scaling dimensions, the central charge $c$ and the parameter $K$ of the sine-Gordon model.

\subsection{The BKT transition point in lower $\lambda$}

The BKT transition point between the non-degenerate ground state phase and the BKT critical phase is given by the level crossing of the following energy gaps [7]:

$$
\begin{aligned}
x_{2,0} & =\frac{L}{2 \pi v} \Delta E(Q=2, \mathrm{PBC}), \\
x_{0, \frac{1}{2}} & =\frac{L}{2 \pi v} \Delta E(Q=0, \mathrm{TBC}, C=1) .
\end{aligned}
$$




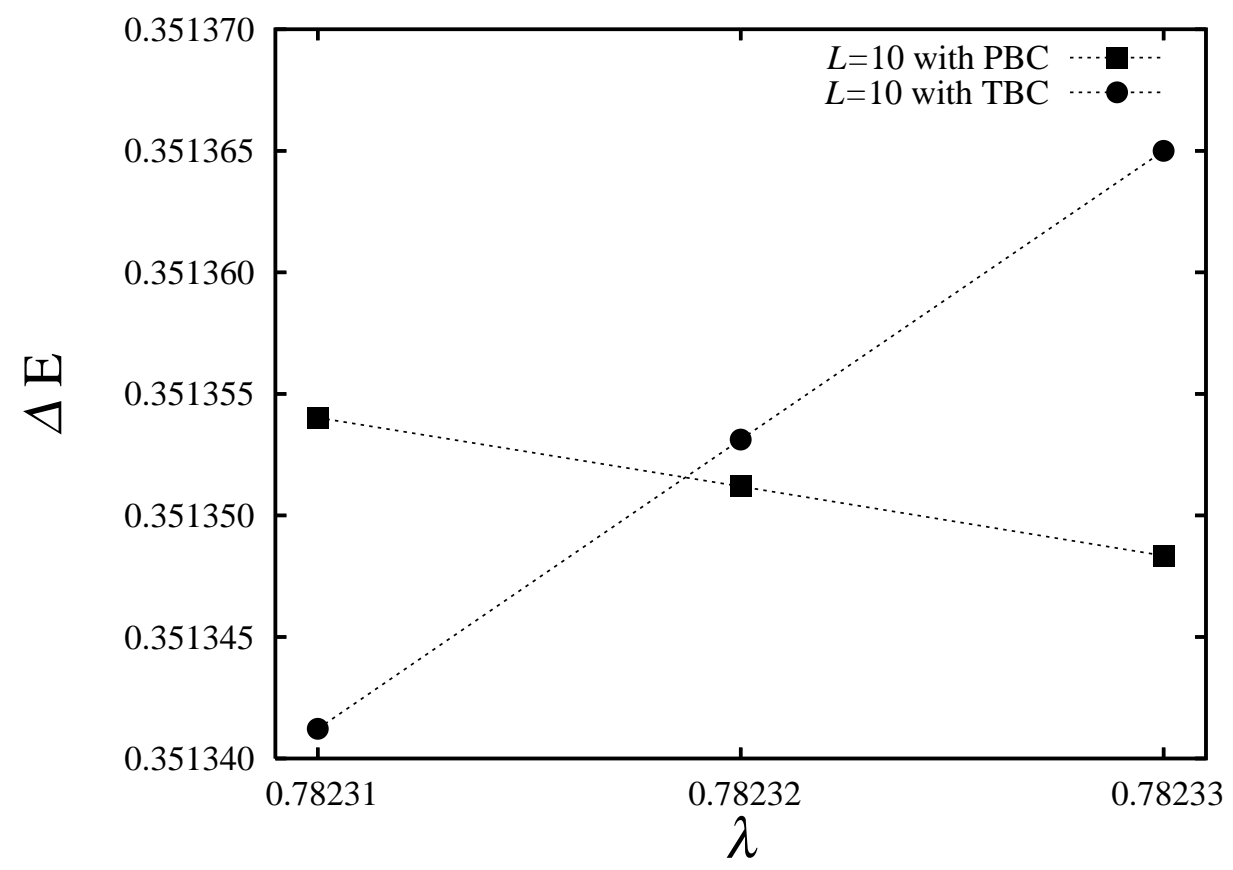

Figure 1: The crossing point for $L=10$ in lower $\lambda$

$\Delta E$ is an energy gap between the ground state and the excitation state with the quantum number $Q$ and a periodic boundary condition(PBC) or a twisted boundary condition(TBC). In terms of the toroidal boundary condition(5), PBC corresponds to $\tilde{Q}=0$, and TBC corresponds to $\tilde{Q}=p / 2$. The ground state has $Q=0, C=1$ with $\mathrm{PBC}$.

Figure shows the crossing point for $L=10$. On the BKT transition point, we can eliminate logarithmic corrections including higher terms by the level spectroscopy, but still we have finite size corrections due to the irrelevant field $L_{-2} \bar{L}_{-2} \mathbf{1}(x=4)$ [13] which behaves order of $1 / L^{2}, 1 / L^{4}$, etc. In figure 2, we show the extrapolation of the level crossing as $L$ tends to infinity. The result is $\lambda=0.78183$.

\subsection{The BKT transition point in upper $\lambda$}

The upper BKT critical point between the 6-fold degenerate ground state phase and the critical phase is given by the crossing point of the following 


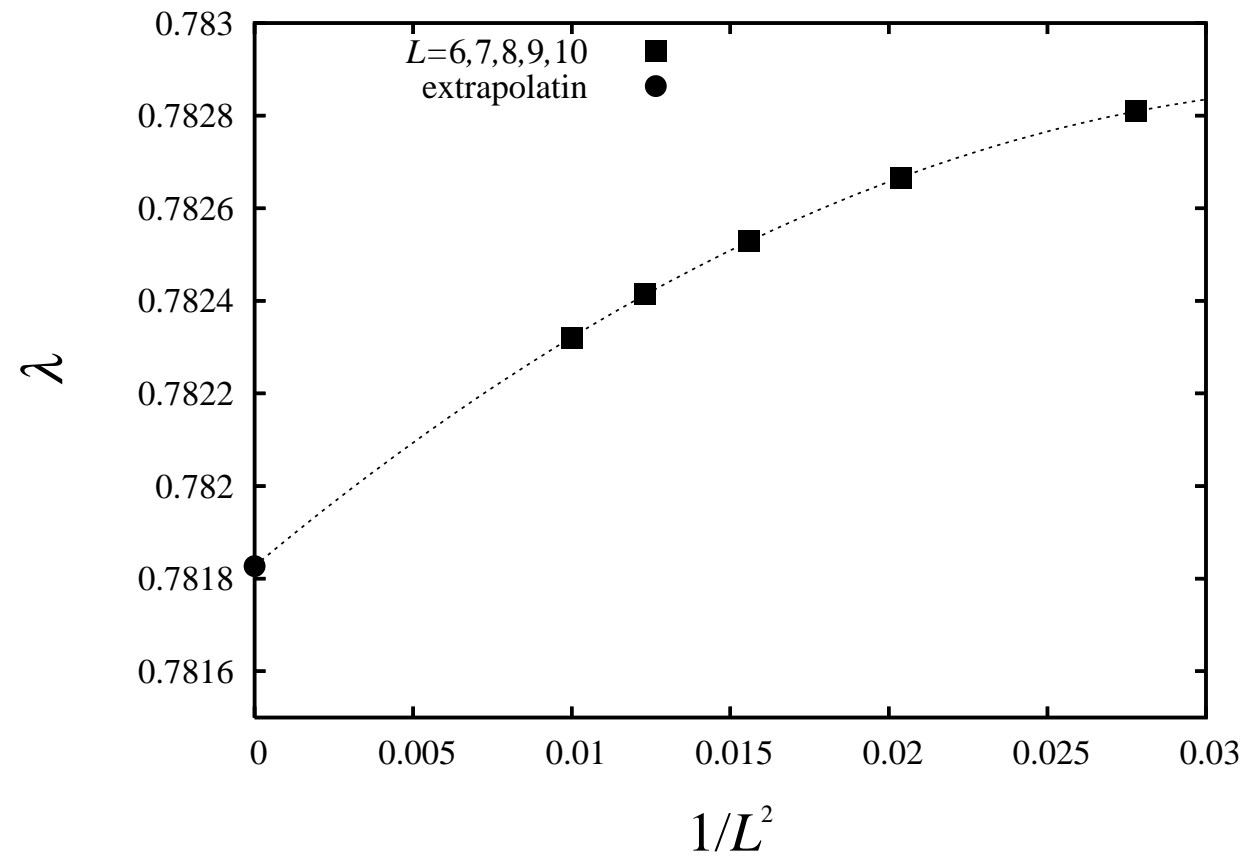

Figure 2: The lower BKT transition point. The extrapolated value is $\lambda=$ 0.78183 . 


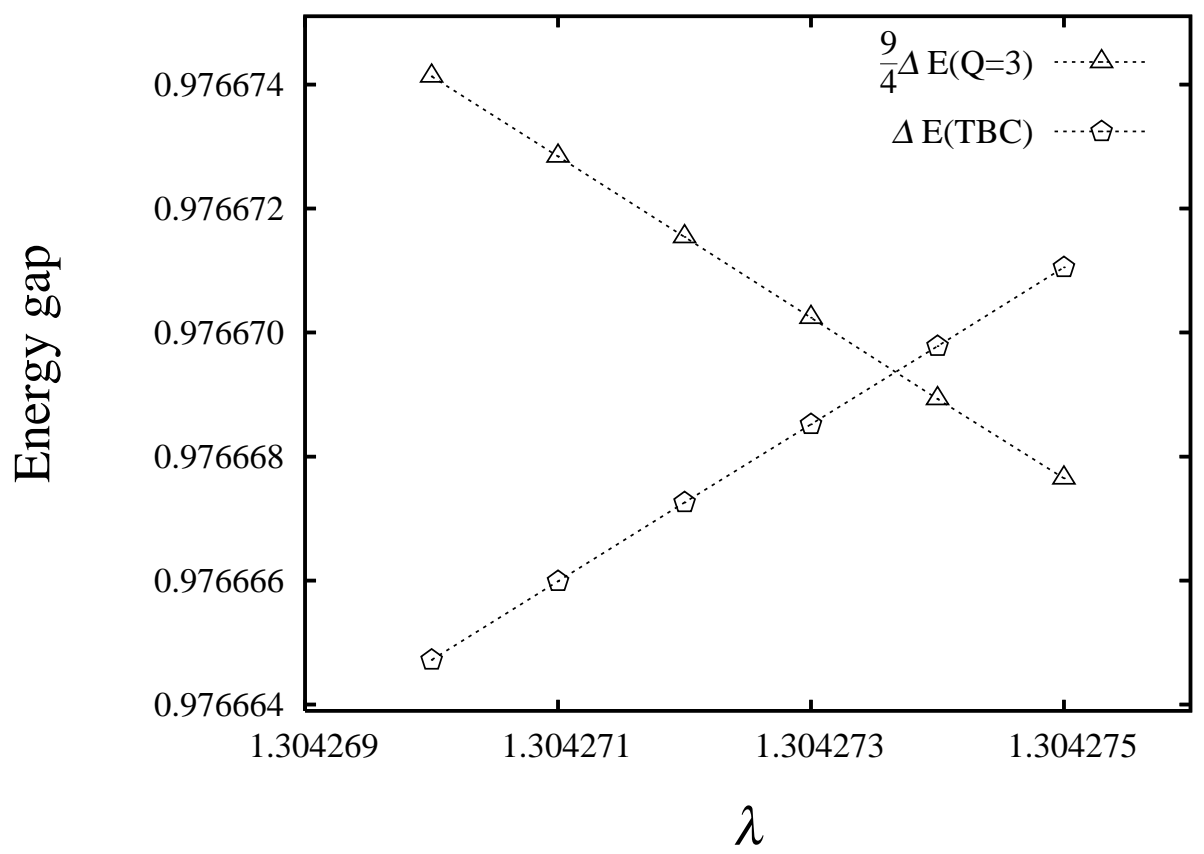

Figure 3: The crossing point for $L=10$ in upper $\lambda$

scaling dimensions $[6][?]$

$$
\begin{aligned}
\frac{9}{4} x_{3,0} & =\frac{9}{4} \frac{L}{2 \pi v} \Delta E(Q=3, P B C, C=1), \\
x_{0, \frac{1}{2}} & =\frac{L}{2 \pi v} \Delta E(Q=0, T B C, C=1) .
\end{aligned}
$$

For $Q=0,3$, the charge conjugation $C$ can be a good quantum number as mentioned at the end of the subsection 2.2.

The crossing point is shown in Figure 3 for $L=10$. This crossing eliminates the logarithmic correction order of $\mathcal{O}(1 / \ln L)$. But still higher order corrections like $O\left(1 /(\ln L)^{2}\right)$ can remain in this case. Anyway we extrapolate

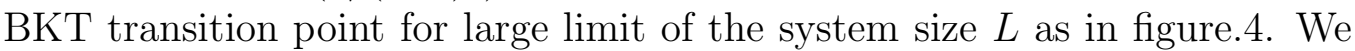
obtain $\lambda=1.2851$.

The product of $\lambda_{\text {upper }}$ and $\lambda_{\text {lower }}$ can be used to check the consistency of the self duality; that is, $\lambda_{\text {upper }} \cdot \lambda_{\text {lower }}$ should be unity. Actually, from the numerical data, we get

$$
\lambda_{\text {upper }} \cdot \lambda_{\text {lower }}=1.0047 .
$$




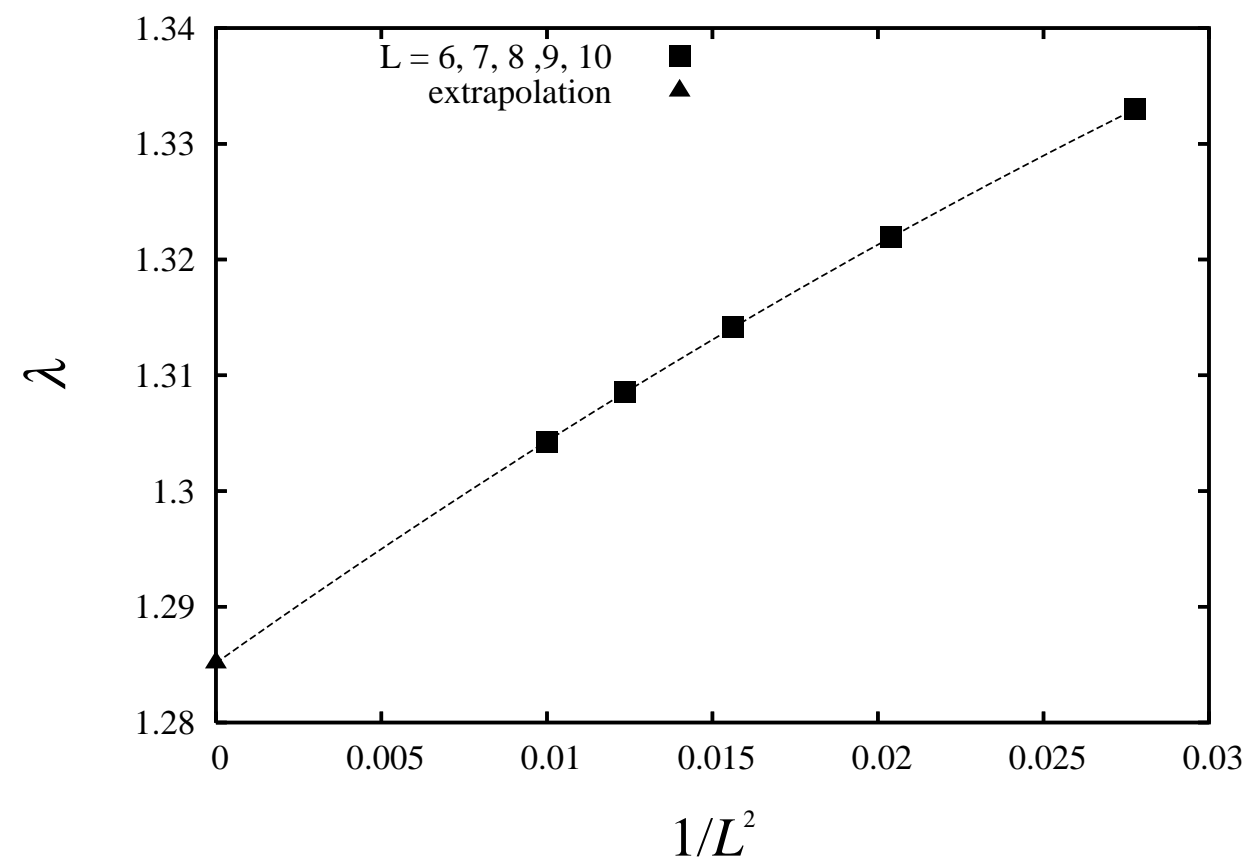

Figure 4: The upper BKT transition point. The extrapolated value is $\lambda=$ 1.2851 . 


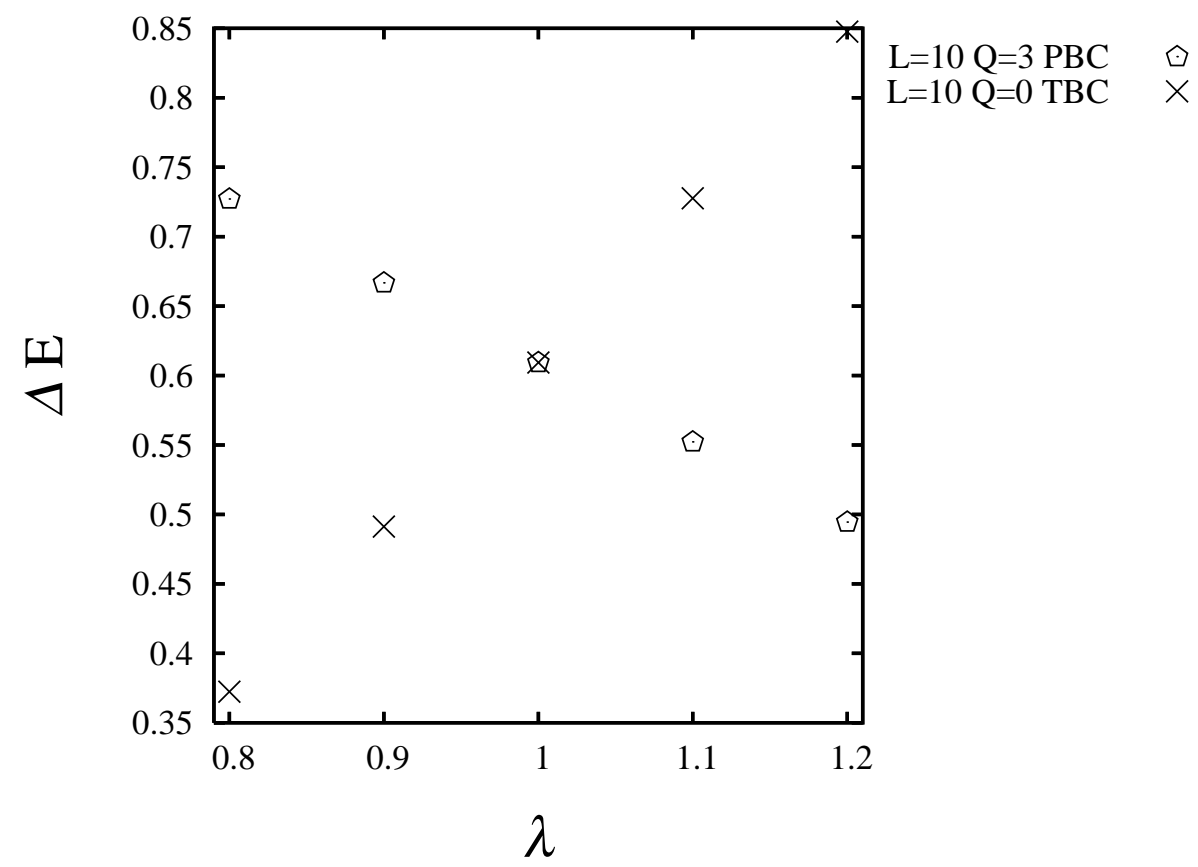

Figure 5: Level crossing occurs exactly at the self-dual point $\lambda=1$. These levels are $\Delta E(Q=3, \mathrm{PBC}, C=1)$ and $\Delta E(Q=0$, TBC, $C=1)$ for $L=10$. The other system sizes also have the exact crossing.

\subsection{Universality class}

\subsubsection{Duality}

We numerically calculate the change of $K$ via $\lambda$ by the ratio of scaling dimensions:

$$
\frac{\Delta E(Q=3, \mathrm{PBC}, C=1)}{\Delta E(Q=0, \mathrm{TBC}, C=1)}=\frac{x_{3,0}}{x_{0, \frac{1}{2}}}=36 / K^{2} .
$$

This ratio has the logarithmic correction $\mathcal{O}\left(1 /(\ln L)^{2}\right)$ in the vicinity of the BKT transition points. But at the self-dual point, $\Delta E(Q=3$, PBC, $C=1)$ and $\Delta E(Q=0, \mathrm{TBC}, C=1)$ exactly degenerate; therefore exactly $K=6$. This is because the dual sine-Gordon model has higher symmetry at the selfdual point as was discussed in (31), (32). We show this in figure 5 . The energy excitations: $\Delta E(Q=3, \mathrm{PBC}, C=1)$ and $\Delta E(Q=0$, TBC, $C=1)$ exactly cross at $\lambda=1$. 


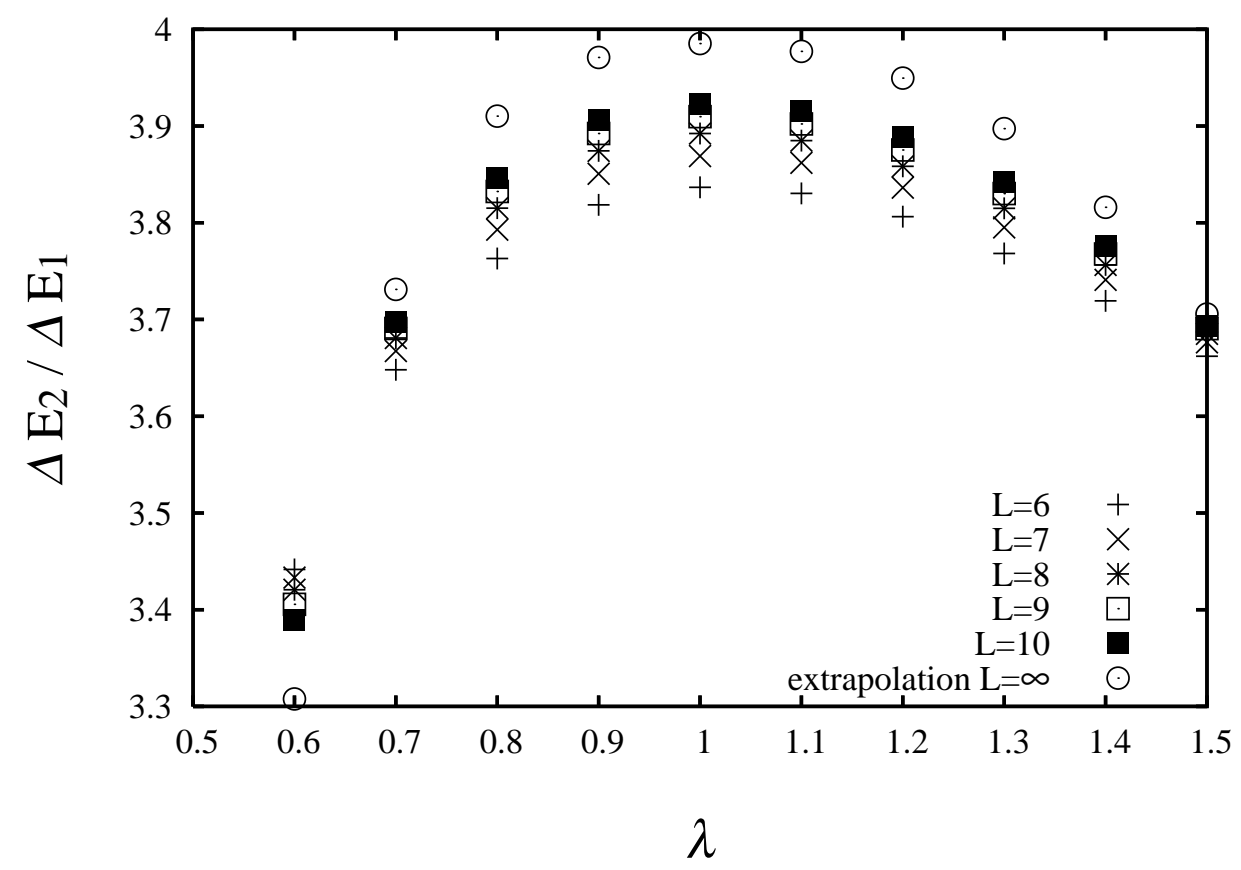

Figure 6: Ratio of scaling dimensions $x_{2,0} / x_{1,0}$. In the critical region the ratio $x_{2,0} / x_{1,0} \simeq 4$

von Gehlen and Rittenberg [15] pointed out that the spectra of the $Z_{p^{-}}$ symmetric quantum chains at the self-dual point are $p$-fold degenerated. Our novel point is not only the degeneracy but the connection of the discrete $Z_{p}$ model and the $Z_{p}$ dual sine-Gordon model.

Even though when one study some other lattice model which doesn't have the self-duality explicitly, but if it could be mapped to the dual sineGordon model, one can check the self-dual point of the dual sine-Gordon model through this level crossing. For example, such an approach will be useful in the vicinity of the multicritical point studied by Otsuka et al[?].

\subsubsection{Ratio of scaling dimensions}

The ratio of scaling dimensions

$$
\frac{x_{2,0}}{x_{1,0}}=\frac{\Delta E(Q=2, P B C)}{\Delta E(Q=1, P B C)}=4+\left(L^{-2}\right)+\text { higherorder }
$$


is also useful to check the universality class of the BKT critical region. The finite correction behaves order of $\mathcal{O}\left(L^{-2}\right)$. Figure 6 shows the ratio.

\subsubsection{Central charge}

Generally, in a BKT critical region a renormalization equation flows to the Gaussian fixed line. Therefore the universality class of the BKT critical region agrees with the Gaussian model. The Gaussian model is known to have the central charge $c=1$, so the BKT critical region is also characterized by $c=1[16$.

From the conformal field theory the central charge is related to the ground state of the finite system as 17]

$$
E_{g}(L)=e_{g} L-\frac{\pi v c}{6 L}\left(1+\mathcal{O}\left(\frac{1}{(\ln L)^{3}}\right)\right)
$$

where $e_{g}$ is a free energy per site and $v$ is a velocity of the system. This is one of the important result of the conformal field theory. In this case the logarithmic correction is small enough, so the central charge is numerically a good index of universality class.

We calculate the effective central charge for the system sizes up to $L=10$ and extrapolated to $L \rightarrow \infty$. In Figure [1, we show the extrapolated values of the central charge. This result is consistent with the two transition points we have determined.

\section{Conclusion}

We extended the level spectroscopy to be able to determine the BKT critical point between the multi-fold degenerated phase and the critical region. For a physical application we studied the 1D quantum $p$-clock model which has the $Z_{p}$ symmetry. The numerical calculations were performed for $p=6$. And also we discussed about the self-duality of the dual sine-Gordon model. In the section III-C, the self-dual point could be numerically determined without logarithmic corrections. This is because the level crossing of $\Delta E(Q=p / 2, \mathrm{PBC})$ and $\Delta E(Q=0, \mathrm{TBC})$ is the exact result from the duality(11). There is no correction term in the langage of the dual sine-Gordon model (19). On the other hand, the BKT transition point that we determined have some correction beause we ignore irrelevant terms to derive (25) and (26) from (19). Our 


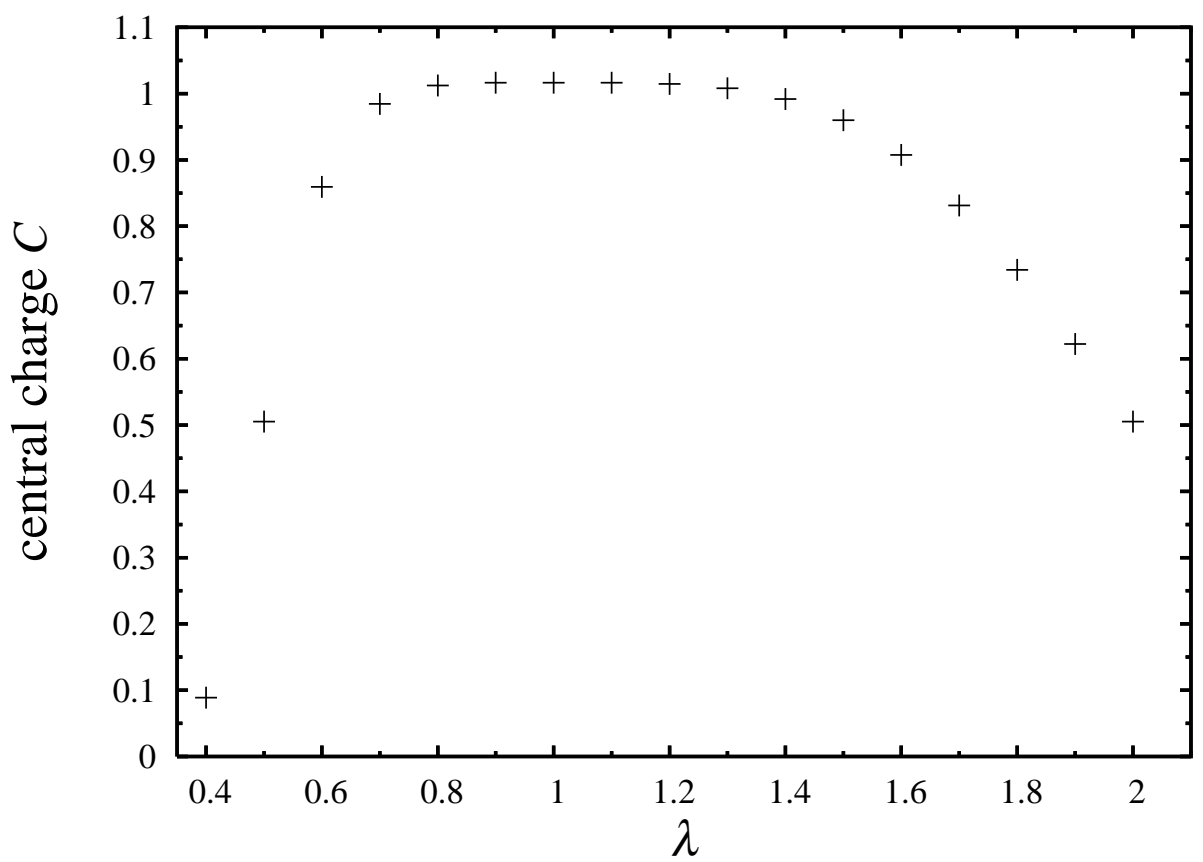

Figure 7: The central charge extrapolated from the finite system's results. 
present approach will be useful for the other models which need more highly accurate calculations, for instance the cross-over near the multicritical point dealed in [?].

\section{References}

[1] Berezinskii Z L. Zh. Eksp. Teor. Fiz., 61:1144, 1970.

[2] Kosterlitz J M and Thouless D J. J. Phys. C: Solid State Phys, 6:1181, 1973.

[3] José J V, Kadanoff L P, Kirkpatrick S, and Nelson D R. Phys.Rev.B, $16: 1217,1977$.

[4] Elitzur S, Pearson R B, and Shigemitsu J. Phys. Rev. B, 19:3698, 1979.

[5] Nomura K and Okamoto K. J. Phys A: Math. Gen., 27:5773, 1994.

[6] Nomura K. J. Phys A: Math. Gen., 28:5451, 1995.

[7] Nomura K and Kitazawa A. J. Phys A: Math. Gen., 31:7341, 1998.

[8] Tonegawa T, Okamoto K, Okunishi K, Nomura K, and Kaburagi M. Physica B, 346:50, 2004.

[9] Otsuka H, Mori K, Okabe Y, and Nomura K. Phys. Rev. E, 72:046103, 2005.

[10] Sólyom J and Pfeuty P. Phys. Rev. B, 24:218, 1981.

[11] Destri C. Phys. Rev. B, 223:365, 1989.

[12] Wiegmann P B. J. Phys. C: Solid state Phys., 11:1583, 1978.

[13] Cardy J L. Nucl. Phys. B, 270:186, 1986.

[14] Affleck I. Phys. Rev. Lett., 56:746, 1985.

[15] von Gehlen G and Rittenberg V. Nucl. Phys. B, 257:351, 1985.

[16] Haldane F D M. J. Phys. C: Solid state Phys., 14:2585, 1981.

[17] Belavin A A, Polyakov A M, and Zamolodchikov A B. Nucl. Phys. B, B241:333, 1984. 